\title{
ESTRATEGIAS TECNOLÓGICAS EMPLEADAS EN LA EXPLOTACIÓN DE MATERIAS PRIMAS Y FORMATIZACIÓN DE ARTEFACTOS EN PAMPA OESTE ZONA DE APROVISIONAMIENTO Y CANTERA (ANTOFAGASTA DE LA SIERRA - CATAMARCA, ARGENTINA) ${ }^{1}$
}

\author{
TECHNOLOGICAL STRATEGIES USED IN THE EXPLOITATION OF RAW \\ MATERIALS AND THE KNAPPING OF TOOLS IN PAMPA OESTE ZONA DE \\ APROVISIONAMIENTO Y CANTERA \\ (ANTOFAGASTA DE LA SIERRA - CATAMARCA, ARGENTINA)
}

\author{
Federico Miguel Bobillo ${ }^{2}$
}

\begin{abstract}
La cantera de vulcanitas denominada Pampa Oeste Zona de Aprovisionamiento y Cantera (POZAC) presenta evidencias de actividades de talla orientadas a la extracción de recursos líticos, los cuales se transportaron a campamentos y bases residenciales situados en las localidades de Quebrada Seca y Punta de la Peña (Antofagasta de la Sierra - Catamarca, Argentina). La presente investigación tuvo por objeto estudiar los contextos líticos de las áreas de talla que conforman POZAC, analizando los criterios que tuvieron los talladores al momento de seleccionar los recursos líticos a explotar, como así también los métodos y técnicas empleados en la gestión de bloques, nódulos y lascas nodulares para la extracción de formas-base y la formatización de instrumentos. Para ello se efectuaron prospecciones y muestreos sistemáticos de seis Áreas Discretas de Talla (ADT), junto a un análisis técnico-tipológico de núcleos, nucleiformes, desechos de talla y artefactos formatizados hallados en los contextos de cantera. A partir de esta investigación se pudo constatar la selección, recolección, transporte y reducción de nódulos y bloques de vulcanitas con diferentes tamaños y morfologías, el empleo de lascas nodulares como nucleiformes y la extracción de formas-base con dimensiones variables para la manufactura de instrumentos.
\end{abstract}

Palabras claves: aprovisionamiento de recursos líticos, métodos y técnicas de talla, cantera de vulcanita, análisis

técnico-tipológico.

The volcanic rock quarry Pampa Oeste Zona de Aprovisionamiento y Cantera (POZAC) presents evidence of knapping activities related to the extraction of lithic resources which were transported to camps and residential bases located in Quebrada Seca and Punta de la Peña (Antofagasta de la Sierra - Catamarca, Argentina). The aim of this research was to study the lithic contexts of the knapping areas located at POZAC, by analyzing the selection criteria for the exploitation of these lithic resources as well as the methods and techniques used in the management of blocks, nodules and nodular flakes employed as blanks. For this purpose, we developed systematic surveys and sampling of six "Discrete Knapping Areas" (DKA) and performed techno-typological analyses of cores, core-flakes, flakes and knapping tools recovered. It was possible to establish the characteristics of selection, collection, transportation and reduction of nodules and blocks of volcanic rocks with different sizes and morphologies, the usage of nodular flakes as core-flakes, and the extraction of blanks of variable dimensions for knapping tools.

Key words: Lithic resources procurement, knapping methods and techniques, volcanic rock quarry, technical-

typological analysis.

Las poblaciones prehispánicas desarrollan distintas estrategias tecnológicas en torno al aprovisionamiento de recursos líticos, dependiendo de las necesidades que surgen como parte de las actividades de subsistencia (Haury 1994; Nelson
1991). Estas estrategias implican un orden secuencial o terminal de producción, de acuerdo al tipo de actividades de talla que se desarrollan en las canteras, talleres de manufactura de instrumentos y sitios residenciales y/o logísticos (Ericson 1984).

\footnotetext{
1 Una primera versión de este artículo fue presentada en el simposio "Desde las fuentes: estudios de selección y aprovisionamiento de minerales. Implicancias en la producción lítica, alfarera, metalúrgica y de manifestaciones rupestres", en el marco del XIX Congreso Nacional de Arqueología Argentina, Tucumán (agosto 2016). Este manuscrito fue evaluado por pares externos y editado por el Comité Editorial de Chungara y los editores invitados Ariel D. Frank, Fabiana Skarbun y Manuel E. Cueto.

2 Instituto Superior de Estudios Sociales-CONICET, San Lorenzo 429 (CP 4000), San Miguel de Tucumán, Tucumán, Argentina. fede_bobillo@yahoo.com.ar
}

Recibido: febrero 2017. Aceptado: enero 2018.

http://dx.doi.org/10.4067/S0717-73562018005000302. Publicado en línea: 5-marzo-2018. 
Particularmente, en los sitios cantera, la explotación de materias primas se lleva a cabo a través de distintas estrategias de aprovisionamiento, entre las que se pueden considerar: el canteo (excavación de pozos o túneles con el fin de extraer el recurso), extracción (remoción de materia prima de las fuentes expuestas) y el aprovisionamiento oportuno (realizado desde depósitos secundarios y/o terciarios) (Church 1995; Haury 1994). Dichos procedimientos implican la caracterización del recurso que va a ser explotado (selección), la extracción de bloques de rocas, reducción y formatización de productos terminados o parcialmente terminados (producción), y el transporte de los mismos dentro y fuera de la cantera (logística) (Bloxam y Heldal 2008).

En el Noroeste argentino, se han desarrollado distintos estudios sobre el aprovisionamiento de recursos líticos, los cuales aportaron información sobre las características de los materiales tallados en las canteras-taller, como así también sobre las etapas del sistema de producción representadas en las mismas; los eventos de uso y re-uso del paisaje y la temporalidad de las actividades de talla (Carbonelli 2015; Somonte 2005; Somonte y Baied 2011; entre otros). Específicamente, en la microrregión de Antofagasta de la Sierra se desarrollaron investigaciones que tuvieron por objeto estudiar la organización de la tecnología lítica y los sistemas de producción en sitios cazadores-recolectores y agro-pastoriles datados entre el 10.000 y el 1.000 a.p. aproximadamente, abordando temas como la distribución de las fuentes de aprovisionamiento y diversidad de materias primas explotadas, las etapas del sistema de producción desarrolladas en las canteras y su vínculo con las actividades de confección de toolkits documentadas en sitios residenciales, campamentos, talleres y espacios de caza (Aschero et al. 2002-2004; Escola 2002; Funes Coronel y Martinez 2013; Hocsman 2006; Martinez 2003; Pintar 1996; Toselli 1998; entre otros).

En el marco de dichas investigaciones se pudo establecer un sector de proveniencia de los recursos líticos empleados para la confección de instrumentos, definido como Pampa Oeste (Aschero 1986, 1988; Aschero et al. 2002-2004; Escola 2002; Martinez 2003; Pintar 1996). Zonas de Aprovisionamiento y Cantera (ZAC), como Pampa Oeste, son sitios donde yacen grandes bloques y nódulos de vulcanitas de buena calidad para la talla. Asimismo, se caracterizan por numerosos episodios de extracción y formatización de recursos; con un registro lítico que evidencia procedimientos de reducción de núcleos, extracción de formas-base y primeros estadios de adelgazamiento bifacial de bifaces (Aschero 1988).

En función de lo expresado, la presente investigación tiene por objeto profundizar los estudios en Pampa Oeste -a la que se referirá como Pampa Oeste Zona de Aprovisionamiento y Cantera (POZAC)-, situada entre las localidades de Punta de la Peña y Quebrada Seca, en el curso medio-superior del río Las Pitas (Figura 1-a). Interesa efectuar una aproximación a los criterios que los talladores emplearon para seleccionar el recurso lítico a explotar y los métodos de talla que se habrían utilizado, de acuerdo a los atributos morfológicos y dimensionales de los bloques/nódulos de vulcanitas. También se busca profundizar el conocimiento sobre el proceso de producción dentro de la cantera. Este último abarca el conjunto de actividades que van desde la explotación de núcleos de vulcanitas y la selección y transporte de formas-base hasta la formatización final o parcial de instrumentos dentro de la cantera (Bloxam y Heldal 2008; Doelman 2005; Ericson 1984; Funk 2004). Dicha evidencia se interpreta en función de los hallazgos realizados en sitios residenciales y logísticos de Punta de la Peña y Quebrada Seca, con el fin de aportar datos sobre la temporalidad de las actividades de talla en POZAC.

\section{Antecedentes de Investigaciones Arqueológicas en Pampa Oeste Zona de Aprovisionamiento y Cantera (POZAC)}

Como punto de partida, es preciso considerar que POZAC es una fuente secundaria de materias primas (sensu Nami 1992). Esta se caracteriza por presentar clastos transportables y no transportables de tamaño guijón y bloque (González Bonorino y Teruggi 1952), aislados obien agrupados determinando zonas de mayor concentración (Bobillo y Hocsman 2015). Dichos materiales fueron explotados por las poblaciones prehispánicas con el propósito de extraer lascas de gran tamaño para ser usadas como formas-base de artefactos formatizados, entre ellos bifaces -considerando la evidencia en la cantera de los primeros estadios de reducción bifacial-, o bien para emplearse como otros tipos de núcleos (Aschero 1986, 1988; Aschero et al. 2002-2004; Pintar 1996).

Por su parte, los conjuntos artefactuales distribuidos en la superficie de la cantera se caracterizan por formar Áreas Discretas de Talla (ADT). Estas pueden ser entendidas como "locus de talla"; caracterizadas por conjuntos de núcleos, nucleiformes, lascas, artefactos formatizados y percutores, que se presentan en concentraciones 


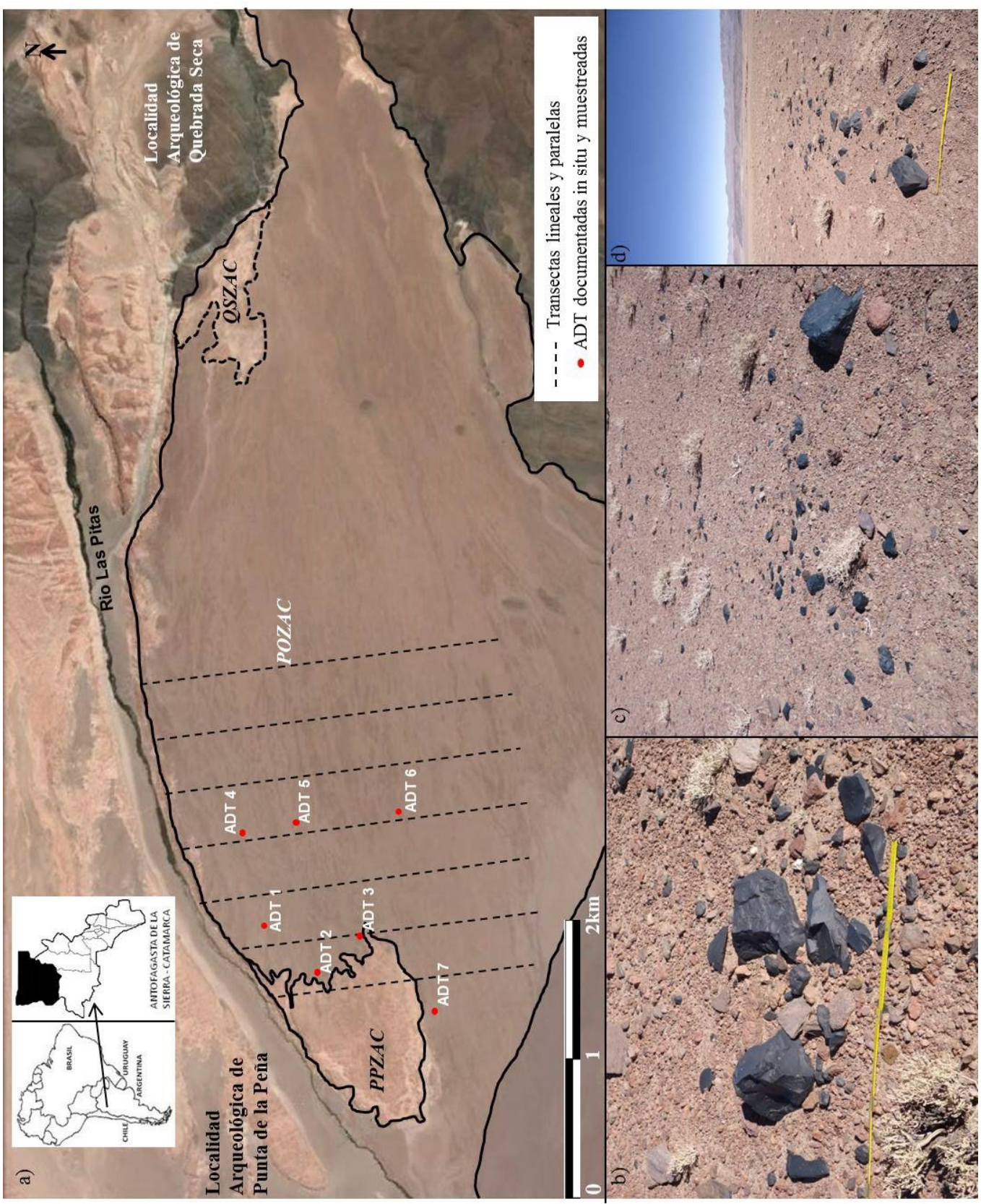


poco densas y variables, de 4 a $10 \mathrm{~m}$ de diámetro (Figura 1-b y 1-c). Además, poseen una distribución irregular sobre el pedimento y sin asociación espacial de unas con otras, dependiendo de cómo yacen los bloques de grandes dimensiones y clastos de vulcanita sobre la superficie de la cantera (Bobillo 2017). Investigaciones realizadas en el Área Discreta de Talla 1 (ADT 1) (Bobillo 2015, 2017), situada en el sector occidental de POZAC (Figura 1-a), lograron determinar una tendencia hacia la explotación de bloques/nódulos con dimensiones que van desde los $12 \mathrm{~cm}$ hasta los 28 $\mathrm{cm}$ (Bobillo 2015). Los negativos de lascados de dichas piezas -particularmente núcleos poliédricos y de lascados aislados- muestran una tendencia a la extracción de formas-base de gran porte, es decir, lascas muy grandes y anchas (sensu Aschero 1975, 1983).

Por otra parte, Aschero (1986) menciona que los instrumentos aparecen escasamente representados en los conjuntos artefactuales de POZAC, observándose bifaces espesos con aristas poco regularizadas que podrían ser núcleos bifaciales. En ADT 1, se registraron artefactos confeccionados por retoque marginal, aunque no se logró documentar la presencia de actividades de talla orientadas al adelgazamiento bifacial de formas-base (Bobillo 2017). Estudios previos postularon que algunas etapas de la reducción bifacial se podrían haber realizado en las ADT de POZAC, pero las piezas no habrían sido terminadas allí (Aschero 1986, 1988; Pintar 1996).

En función de los antecedentes mencionados, en el presente estudio se busca profundizar el conocimiento sobre los procesos de selección, producción y logística desarrollados por los individuos y grupos de individuos como parte de las actividades de aprovisionamiento en POZAC, abordando los atributos técnico-tipológicos de los conjuntos líticos registrados y muestreados en las ADT.

\section{Metodología: Prospecciones Sistemáticas, Muestreos y Análisis Técnico-Tipológico de Conjuntos Líticos}

El área de cantera se presenta como una extensa planicie de ignimbrita que se asocia a la caldera del Cerro Galán y se encuentra recubierta por el nivel de pedimentación II (Tchilinguirian 2008). Los pedimentos son superficies de erosión, de pendientes muy suaves y con poca vegetación, con gradientes de $3^{\circ}$ a $6^{\circ}$, que están cubiertos por una delgada capa de conglomerados. Presentan una superficie plana, cubierta por pavimento del desierto (Figura 1-d) (Tchilinguirian comunicación personal 2014 en Bobillo y Hocsman 2015).

Considerando dichas características del terreno, se escogió una estrategia de recorrido que permitiera sistematizar el registro de las ADT y recabar información sobre su distribución y composición artefactual. Para ello, se realizaron transectas lineales y paralelas, con el fin de localizar estos eventos de talla y registrar sus dimensiones y las características técnico-tipológicas de los conjuntos líticos. Una vez identificadas las ADT del sector occidental se seleccionaron seis (ADT 2, ADT 3, ADT 4, ADT 5, ADT 6 y ADT 7) (Figura 1-a) para muestrear y efectuar un análisis técnico-tipológico (Aschero 1975, 1983; Aschero y Hocsman 2004; Bellelli 1991). ADT 1 se analizó en investigaciones previas (Bobillo 2015, 2017) y sus datos se retoman en la discusión del presente artículo.

Particularmente, el análisis de las ADT implicó un registro in situ de las características técnicotipológicas de núcleos no transportables (bloques usados cono núcleos con dimensiones superiores a los 35 o $40 \mathrm{~cm}$ y pesados) y desechos de talla. Además, se efectuaron muestreos de núcleos (transportables), nucleiformes (lascas nodulares usadas como núcleos para extraer otro tipo de lascas), artefactos formatizados y materiales tipológica/temporalmente sensibles (bifaces en proceso de manufactura).

Por su parte, en ADT 2, se realizó un muestreo de dos por dos metros con el fin de obtener una muestra de conjuntos líticos completos, y dos sondeos de un metro por un metro para corroborar si este sector de la cantera poseía o no potencia estratigráfica. El muestreo en ADT 2 se realizó sobre un sector de mayor densidad de materiales líticos, con presencia de núcleos, nucleiformes, desechos de talla y artefactos formatizados. Las dimensiones del muestreo se establecieron en función de obtener una muestra representativa para su posterior análisis en el laboratorio.

Las ADT seleccionadas para efectuar muestreos se escogieron considerando los siguientes criterios: (1) presencia de registro lítico que manifestara variabilidad en los procedimientos de reducción de núcleos y extracción de formas-base y (2) presencia y diversidad de artefactos formatizados (terminados o en proceso de manufactura) asociados a eventos de extracción de vulcanitas. Estos últimos se estudian con el objeto de recabar información sobre los procedimientos de confección de toolkits que los talladores desarrollaron en contextos de aprovisionamiento. Además, se muestrearon materiales tipológica y temporalmente sensibles (bifaces en proceso de manufactura) con el fin de 
establecer comparaciones con las evidencias de ocupaciones de sitios residenciales de la localidad de Punta de la Peña y Quebrada Seca.

\section{Resultados}

Las ADT relevadas en el sector occidental de POZAC representan eventos de reducción de núcleos para la extracción de formas-base, observándose algunos contextos donde se habrían desarrollado actividades de formatización de artefactos. A partir de los sondeos realizados en ADT 2 se logró corroborar que posee escasa potencia sedimentaria de arenas poco consolidadas (15 cm aproximadamente), por lo que se trataría de un taller superficial. En la Tabla 1 se hace referencia a las clases tipológicas presentes en ADT 2, ADT 3, ADT 4, ADT 5, ADT 6 y ADT 7. Los atributos técnico-tipológicos de los conjuntos líticos se detallan en el siguiente apartado.

\section{Atributos técnico-tipológicos de los conjuntos líticos}

\section{Núcleos y nucleiformes}

Los núcleos mayormente representados en los conjuntos líticos son de lascados aislados (35\%), poliédricos $(29 \%)$, bifaciales de lascados alternantes $(10 \%)$ y nucleiformes (lascas nodulares usadas como núcleos para extraer otro tipo de lascas) $(18 \%)$. En menor proporción se registra un núcleo unifacial de extracciones bidireccionales que parten de dos plataformas opuestas (2\%) y tres fragmentos de núcleos no diferenciados (6\%) (Figura 2). Las materias primas seleccionadas para la explotación son vulcanitas ${ }^{1}$ variedades $1(94 \%)$ y $5(6 \%)$.

En relación a las formas-base de los núcleos y nucleiformes se observa que se emplean distintos tipos de materiales a partir de los cuales se extraen lascas. Con mayor frecuencia se explotan nódulos rodados o facetados (45\%), nódulos tabulares (10\%), nódulos no diferenciados (6\%), lascas angulares con reserva de corteza $(21 \%)$ y sin reserva de corteza (6\%); lascas primarias (6\%), secundarias (2\%) y no diferenciadas con reserva de corteza (2\%). El porcentaje restante corresponde a un artefacto formatizado reciclado como núcleo.

Cabe aclarar que se registra un núcleo de lascados aislados y un nucleiforme sobre lascas de esquina u hombro de núcleo. Estas últimas, podrían haber sido generadas en procedimientos de búsqueda de superficies aptas (frentes de extracción útiles o plataformas de percusión adecuadas) en los bloques y/o nódulos que son reducidos (Goren-Inbar et al. 2011) (Figura 2-f).

Por su parte, las dimensiones de los núcleos y nucleiformes varían (Tabla 2, Figura 2), observándose un rango de explotación amplio que va desde bloques de gran porte con dimensiones próximas -o superiores- a los $40 \mathrm{~cm}$ (Figura 2-a y 2-b) hasta piezas con tamaños más reducidos (10 cm y menores). Asimismo, se observa que la formas-base extraídas poseen distintos tamaños: muy grandes, grandes, medianas y pequeñas (sensu Aschero 1975, 1983).

\section{Desechos de talla}

En los contextos líticos de las ADT se observan lascas primarias, secundarias, angulares con y sin reserva de corteza, y lascas de reactivación de núcleos. Un análisis detallado realizado sobre 421 desechos de talla $\left(\mathrm{NMD}^{(2)}=356\right.$ sensu Aschero et al. 1993-1994) recolectados en ADT 2 permitió efectuar una aproximación a los materiales descartados durante los procesos de talla (Tabla 3). En relación a las materias primas explotadas en dicha ADT solo se registran las variedades de

Tabla 1. Clases tipológicas representadas en las ADT registradas en POZAC. Typological classes represented in DKA recorded at POZAC.

\begin{tabular}{|c|c|c|c|c|c|c|}
\hline Clases tipológicas & ADT 2 (n) & $\operatorname{ADT} 3(n)$ & ADT $4(n)$ & $\operatorname{ADT} 5(\mathrm{n})$ & ADT $6(n)$ & ADT $7(n)$ \\
\hline Núcleos y nucleiformes & 14 & 5 & 6 & 9 & 6 & 11 \\
\hline Desechos de talla ${ }^{1}$ & $\begin{array}{c}\text { Más de } 300 \\
\text { lascas (aprox.) }\end{array}$ & $\begin{array}{c}\text { Más de } 100 \\
\text { lascas } \\
\text { (aprox.) }\end{array}$ & $\begin{array}{c}\text { Más de } 50 \\
\text { lascas } \\
\text { (aprox.) }\end{array}$ & $\begin{array}{c}\text { Más de } 100 \\
\text { lascas } \\
\text { (aprox.) }\end{array}$ & $\begin{array}{c}\text { Más de } 100 \\
\text { lascas } \\
\text { (aprox.) }\end{array}$ & $\begin{array}{c}\text { Más de } 50 \\
\text { lascas } \\
\text { (aprox.) }\end{array}$ \\
\hline $\begin{array}{c}\text { Artefactos } \\
\text { formatizados }\end{array}$ & 42 & 1 & $\begin{array}{l}\text { No se } \\
\text { registran }\end{array}$ & 2 & 3 & 14 \\
\hline Percutores & $\begin{array}{l}\text { No se } \\
\text { registran }\end{array}$ & $\begin{array}{l}\text { No se } \\
\text { registran }\end{array}$ & $\begin{array}{l}\text { No se } \\
\text { registran }\end{array}$ & 1 & $\begin{array}{l}\text { No se } \\
\text { registran }\end{array}$ & $\begin{array}{l}\text { No se } \\
\text { registran }\end{array}$ \\
\hline
\end{tabular}

${ }^{1}$ En todas las ADT se relevaron lascas externas e internas. En el presente trabajo se estudió los atributos técnico-morfológicos del conjunto de desechos de talla muestreado en ADT 2. 
a)

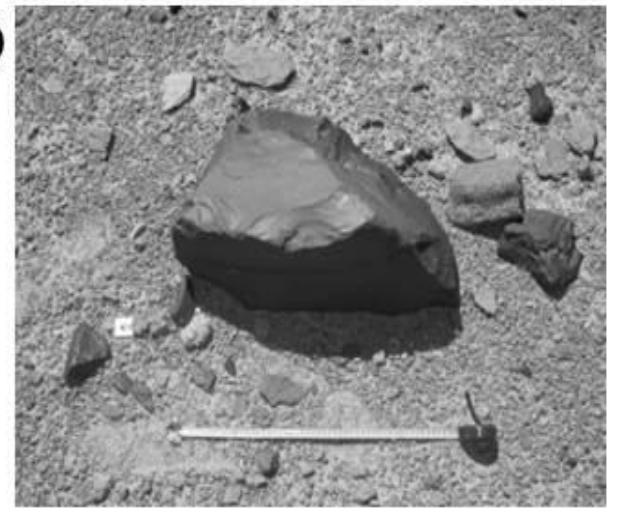

c)

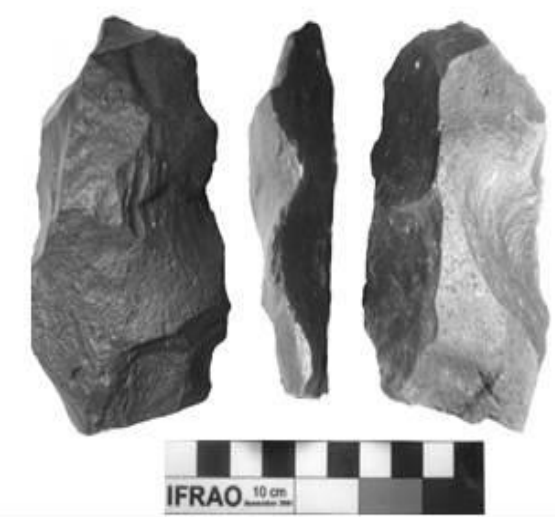

e)

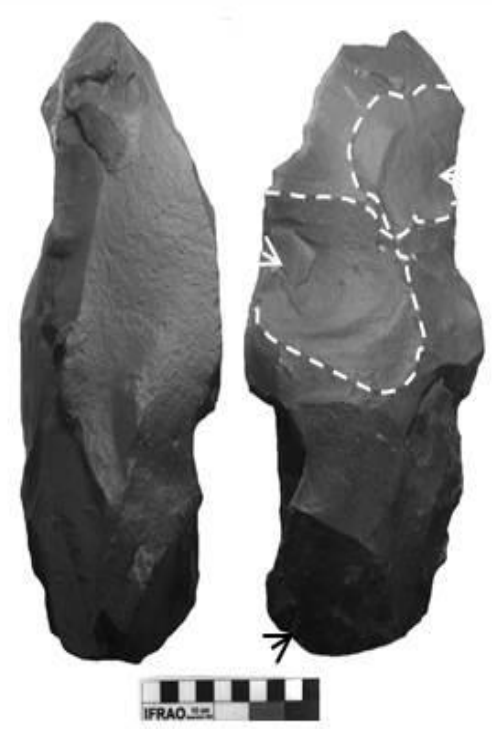

b)

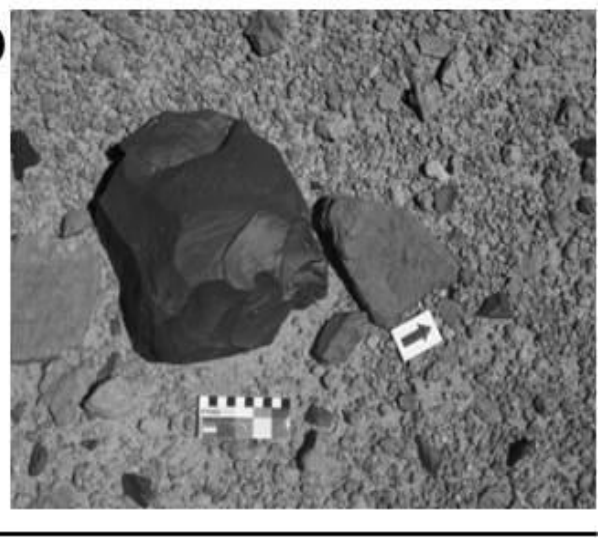

d)

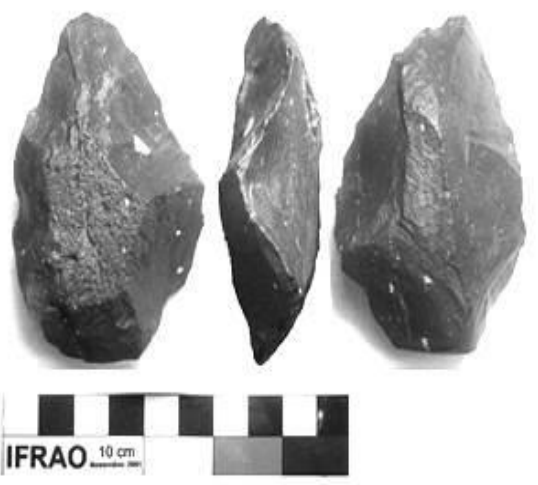

f)
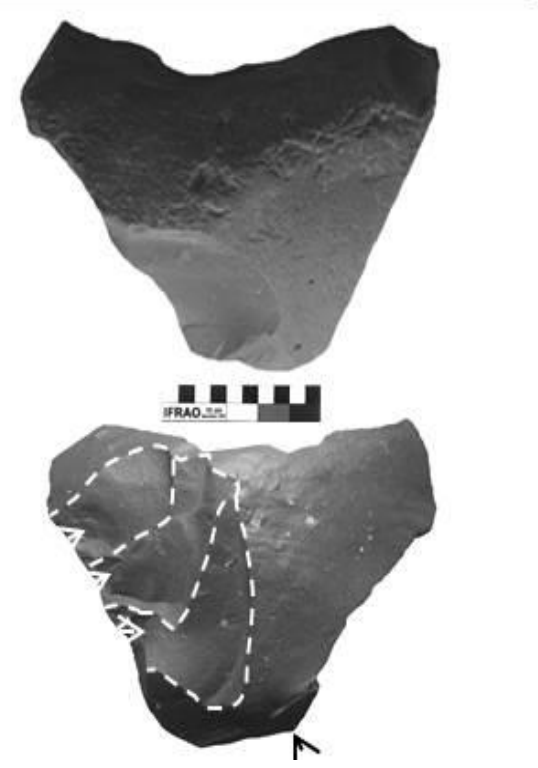

Figura 2. (a y b) Núcleos sobre bloques de vulcanita de grandes dimensiones; (c y d) núcleos bifaciales con dimensiones inferiores a los 30 o $20 \mathrm{~cm}$; (e y f) nucleiformes sobre lascas grandes extraídas de los bloques de vulcanita.

$(a$ and $b)$; Cores on large volcanic blocks; $(c$ and d); bifacial cores with dimensions less than 30 or $20 \mathrm{~cm}$; (e and f); flake cores on large flakes extracted from the blocks of volcanic rocks. 
Tabla 2. Dimensiones promediadas de núcleos/nucleiformes y sus negativos de lascado. Muestra proveniente de las ADT situadas en POZAC. Average dimensions of cores/flake cores and their flake scars. Sample from DKA recorded at POZAC.

\begin{tabular}{ccc}
\hline & $\begin{array}{c}\text { Núcleos con lascados aislados y } \\
\text { poliédricos }(\mathrm{n}=33)\end{array}$ & $\begin{array}{c}\text { Núcleo unifacial de extracción bidireccional, núcleos } \\
\text { bifaciales, y nucleiformes }(\mathrm{n}=15)\end{array}$ \\
\hline Longitudes de núcleos y nucleiformes $(\mathrm{cm})$ & $13,5(33 / 5)^{1}$ & $10,7(22,4 / 3,9)$ \\
Anchos de núcleos y nucleiformes $(\mathrm{cm})$ & $16,4(39 / 4)$ & $10,1(24 / 4,1)$ \\
Espesores de núcleos y nucleiformes $(\mathrm{cm})$ & $4(16 / 2,7)$ & $3,6(9 / 1,6)$ \\
Longitud de la extracción máxima $(\mathrm{cm})$ & $7,7(22 / 3,1)$ & $6,7(8 / 1,9)$ \\
Ancho de la extracción máxima $(\mathrm{cm})$ & $9(23 / 3)$ & $1,7(9 / 1,9)$ \\
Longitud de la extracción mínima $(\mathrm{cm})$ & $4,5(11 / 1,1)$ & $2(8 / 2,5)$ \\
Ancho de la extracción mínima $(\mathrm{cm})$ & $5,7(14 / 2)$ & \\
\hline
\end{tabular}

${ }^{1}$ Entre paréntesis se expresan los tamaños máximos y mínimos (en centímetros), respectivamente, registrados en los núcleos y sus extracciones.

vulcanitas $1(97 \%)$ y 5 (3\%), lo que se condice con lo evidenciado a partir del análisis de núcleos y nucleiformes referido en el apartado anterior. Los desechos de talla provienen de toda la secuencia de reducción (Tabla 3). En el conjunto de lascas primarias y secundarias se documentan lascas de esquina u hombro de núcleos $(n=4)$, extraídas mediante percusión dura y fuerte, dada la presencia de punto de percusión, estrías, bulbos y ondas en las piezas analizadas. Dichas lascas se habrían obtenido con el fin de generar planos y flancos aprovechables en los bloques/nódulos de materia prima (Goren-Inbar et al. 2011).

De acuerdo a las observaciones realizadas, las actividades de reducción se habrían efectuado mediante percusión dura (Whittaker 1994). Prueba de ello es la presencia de lascas con puntos de percusión (80\%), estrías (99\%), bulbos pronunciados (65\%), ondas (97\%) y lascas adventicias (13\%). Asimismo, se llegan a apreciar talones con múltiples marcas de impacto, bulbos de percusión dobles y triples, además de bulbos estallados; lo que evidenciaría el empleo de una fuerza motora intensa y una percusión dura profusa por parte de los talladores para fragmentar bloques de vulcanita y extraer formas-base a partir de ellos.

En cuanto a las dimensiones de las lascas se observan tamaños que promedian $2,9 \mathrm{~cm}$ de largo por $3,1 \mathrm{~cm}$ de ancho y $0,8 \mathrm{~cm}$ de espesor; los talones de las mismas tienen, en promedio, $2 \mathrm{~cm}$ de ancho por 0,6 cm de espesor. Esto sugiere que hubo una tendencia hacia el descarte de lascas pequeñas (sensu Aschero 1975, 1983), las cuales no habrían sido requeridas como formas-base para la confección de toolkits.

\section{Artefactos formatizados}

En las ADT relevadas se registran 62 artefactos formatizados, compuestos por 81 filos y/o puntas (observados en artefactos simples y compuestos), y/o piezas de morfología global (Tabla 4) (Figura 3 ). Entre este conjunto de piezas se registraron dos casos de reciclaje: una raedera reciclada en muesca burilante con filo pasivo formatizado y un fragmento de punta de proyectil reciclado en muesca burilante de retoque alternante.

Las formas-base principalmente utilizadas son lascas generadas durante la secuencia de reducción de núcleos, particularmente angulares (64\%). En relación a los bifaces hallados en ADT 5, ADT 6 y ADT $7(n=4)$ es preciso considerar que los mismos fueron descartados en estadios iniciales de manufactura: dos esbozos de pieza bifacial y dos bifaces parciales (Figura 3-c). Durante el recorrido de las transectas se relevaron diez contextos líticos que poseían bifaces en proceso de manufactura (bifaces parciales). En conjunto, estas piezas podrían formar parte de un toolkit cazador en preparación como los documentados en contextos residenciales de Punta de la Peña y Quebrada Seca, datados entre 8.000 a.p. y 4.300 a.p. aproximadamente (Hocsman 2006; Martinez 2003).

Por su parte, las dimensiones de los instrumentos varían de acuerdo al tipo de forma-base que se seleccionó para la formatización. Por un lado, se evidencia el uso de lascas de gran tamaño -con dimensiones entre 10 y $15 \mathrm{~cm}$ - para la confección de bifaces, cepillos y filos bifaciales de arista sinuosa (Figura 3-b, 3-c, 3-e y 3-f). Además, se registra un raspador de filo corto y un cortante de filo retocado 
Tabla 3. Atributos técnico-morfológicos de lascas de ADT 2 (POZAC).

Technical-morphological attributes of flakes of DKA 2 (POZAC).

\begin{tabular}{lcc}
\hline Origen de las extracciones & NMD & $\%$ \\
\hline Primaria & 10 & 3 \\
Secundaria & 21 & 6 \\
Con dorso natural & 4 & 1 \\
& & 4
\end{tabular}

No diferenciada por fractura/s

con reserva de corteza $\quad 16$

Angular con reserva de corteza $\quad 106 \quad 30$

Angular sin reserva de corteza $\quad 82 \quad 23$

Plana

$\begin{array}{lll}\text { Adelgazamiento bifacial } & 4 & 1\end{array}$

Angular sin diferenciación de

corteza por fractura/s

60

No diferenciada por fractura/s

sin reserva de corteza $\quad 43$

\begin{tabular}{lll} 
Otras internas & 6 & 2 \\
\hline
\end{tabular}

\begin{tabular}{lcc}
\hline Tipos de talones & & \\
\hline Natural (Cortical) & 19 & 5 \\
Liso Natural & 38 & 11 \\
Liso & 142 & 40 \\
Diedro & 33 & 9 \\
Facetado & 29 & 8 \\
Filiforme & 15 & 4 \\
Puntiforme & 2 & 1 \\
No diferenciado por fractura/s & 78 & 22 \\
\hline
\end{tabular}

Número de negativos de

lascado en cara dorsal

\begin{tabular}{lcc}
\hline a 4 & 253 & 71 \\
5 a 9 & 43 & 12 \\
No diferenciado por fractura/s & 60 & 17
\end{tabular}

confeccionados sobre este tipo de lascas. Por otro lado, se observan artefactos confeccionados por retalla y retoque marginal que fueron manufacturados sobre formas-base con dimensiones inferiores a los $10 \mathrm{~cm}$, llegando a promediar $5,1 \mathrm{~cm}$ de longitud, 5,1 $\mathrm{cm}$ de anchura y $1,9 \mathrm{~cm}$ de espesor. Así, se observa que los talladores utilizaron formas-base de distintos tamaños, de acuerdo a la variedad de instrumentos que manufacturaron.
Tabla 4. Grupos tipológicos registrados en las ADT relevadas POZAC. Typological groups registered in sample from DKA located at POZAC.

\begin{tabular}{|c|c|}
\hline Grupo tipológico & $\mathrm{n}=$ \\
\hline Chopper & 1 \\
\hline Bifaz & 4 \\
\hline Filo Bifacial de arista sinuosa & 6 \\
\hline Cepillo & 6 \\
\hline Raspador & 6 \\
\hline Raedera & 5 \\
\hline Cuchillo de filo retocado & 1 \\
\hline Cuchillo de filo natural con dorso formatizado & 2 \\
\hline Cortante de filo retocado & 6 \\
\hline Cortante de filo natural con dorso formatizado & 1 \\
\hline Muesca retocada & 5 \\
\hline Muesca de lascado simple & 5 \\
\hline Denticulado de bisel/oblicuo abrupto de sección asimétrica & 2 \\
\hline Punta entre muescas & 3 \\
\hline Muesca burilante & 4 \\
\hline Buril & 1 \\
\hline Punta burilante & 3 \\
\hline Escoplo & 2 \\
\hline Punta proyectil & 1 \\
\hline Filo formatizado pasivo & 9 \\
\hline Fragmento no diferenciado de rtefacto formatizado & 6 \\
\hline Filo no diferenciado de artefacto formatizado & 2 \\
\hline Total & 81 \\
\hline
\end{tabular}

Sobre la base de la evidencia registrada en los contextos líticos de POZAC se observó que la manufactura de artefactos se llevó a cabo mediante técnicas de adelgazamiento bifacial y por retalla $(55 \%)$ - retoque $(45 \%)$ marginal de filos y/o puntas.

\section{Discusión y Conclusiones}

\section{Estrategias tecnológicas aplicadas a la reducción de bloques y nódulos de vulcanita para la extracción de formas-base}

De acuerdo a los resultados obtenidos en la presente investigación, en POZAC los talladores habrían seleccionado y explotado bloques y nódulos de vulcanita variedad 1, principalmente, 


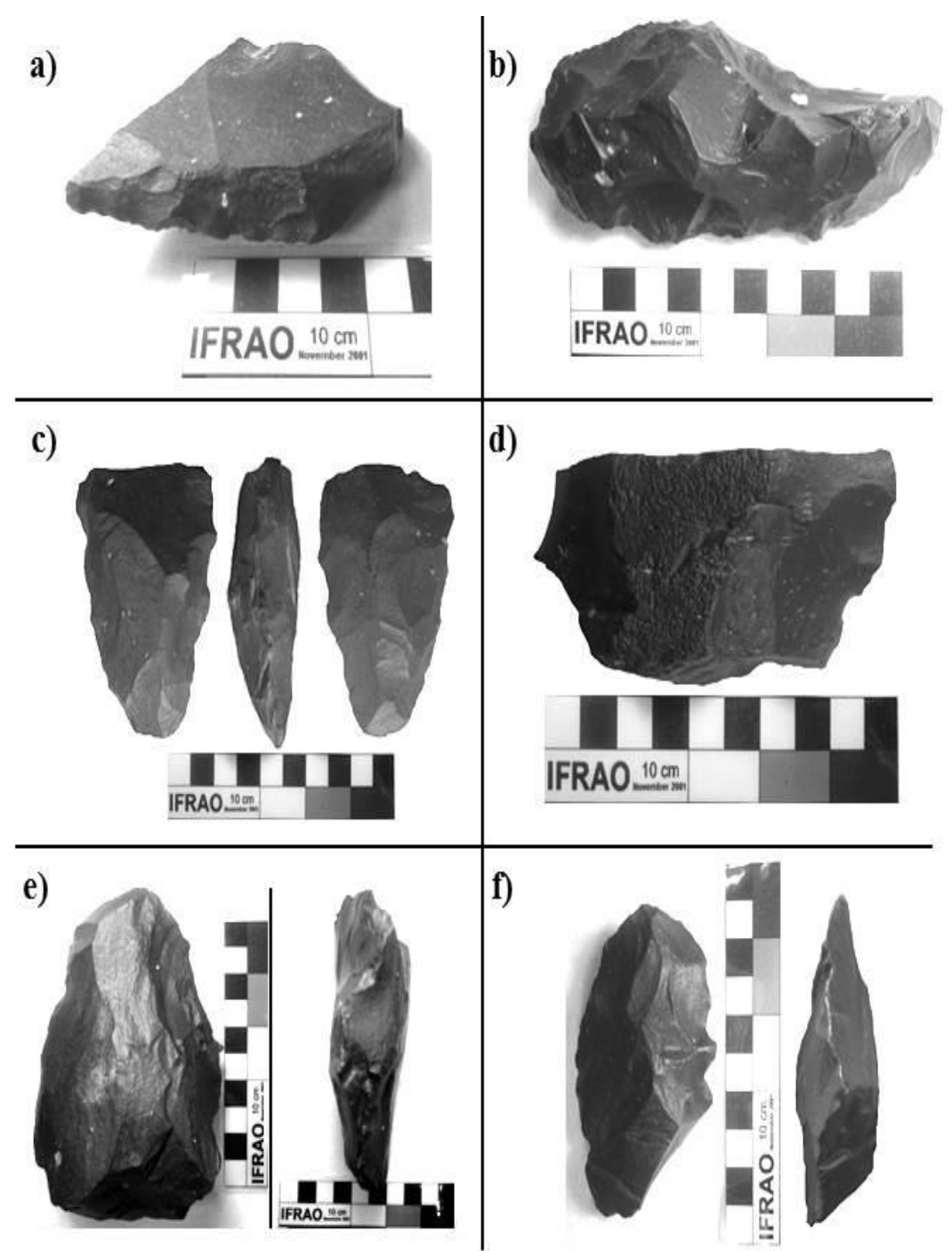

Figura 3. (a) Fragmento de raedera; (b) cepillo; (c) fragmento meso basal de bifaz parcial; (d) chopper; (e y f) filo bifacial de arista sinuosa. (a) Side-scraper fragment; (b) rabot; (c) meso-basal fragment of partial biface; (d) chopper; (e and f) sinuous bifacial edge.

con dimensiones iguales o mayores a $\operatorname{los} 30 / 40 \mathrm{~cm}$. Para ello los talladores emplean un tipo de percusión dura y fuerte que permite reducir bloques y nódulos de vulcanitas. Estudios realizados por Escola (1993) en fuentes de aprovisionamiento de la microrregión de Antofagasta de la Sierra han documentado el uso de percutores con dimensiones próximas a los $10 \mathrm{~cm}$ y pesos entre 1.535 y $885 \mathrm{gr}$ para efectuar las actividades de talla por percusión dura. Fragmentos de percutores hallados en ADT 1 y ADT 5 con tamaños similares a los registrados por Escola (1993), indican una intención de seleccionar piezas grandes y pesadas para impactar y fragmentar grandes masas rocosas.
En función de esta última observación, la reducción de bloques no transportables e inmóviles habría requerido que el tallador desarrolle un tipo de percusión directa arrojando el percutor sobre el objeto de gran tamaño a reducir, o bien impactara la superficie del mismo con un nódulo rodado o facetado de cuarcita o vulcanita sujetado con ambas manos (Colombo 2013; Toth et al. 1992). No se descarta que se hayan aplicado técnicas de reducción de bloque contra bloque (Colombo 2013; Crabtree 1972; Flenniken y White 1985; Toth et al. 1992).

Según lo observado, el tamaño y forma natural de la materia prima habría tenido un estrecho vínculo 
con los métodos y técnicas desarrolladas por los talladores (Flenniken y White 1985), considerando que las superficies naturales de las rocas (facetadas) habrían favorecido la extracción de lascas externas para ser usadas como formas-base. Aschero (1986) postuló que una estrategia de reducción de bloque contra bloque habría permitido realizar extracciones iniciales de fragmentos de nódulos o lascas de gran tamaño, considerando las potencialidades que ofrecían las facetas naturales de los bloques de vulcanita de grandes dimensiones para la extracción de lascas primarias o secundarias (Aschero 1986). Estas formas-base con dimensiones entre 10 y 20 $\mathrm{cm}$ (lascas externas e internas) se habrían empleado para la confección de bifaces e instrumentos con retalla y retoque marginal (cepillos, filos bifaciales de arista sinuosa, entre otros), documentados en las ADT de la cantera. Sin embargo, también se observa una tendencia a la extracción de formas-base con tamaños inferiores a $l o s 10 \mathrm{~cm}$ a partir de bloques de gran porte; como así también desde nódulos y lascas-nucleiformes. Este último conjunto de piezas habrían sido reducidas, posiblemente, mediante una percusión sostenida (Crabtree 1972).

Al considerar los atributos técnico-tipológicos de los núcleos se observan distintos procedimientos para gestionar los recursos líticos como parte de las estrategias de reducción (Inizian et al. 1999). Así, se identifican tipos particulares que se pueden atribuir a diferentes métodos de talla como los descritos por Martín Blanco et al. (1994): talla simple, talla bifacial y talla multifacial. Estos procedimientos se corresponden con la variabilidad de núcleos frecuentemente registrada en las muestras de las ADT de POZAC, particularmente: núcleos de lascados aislados y nucleiformes (de talla simple), núcleos bifaciales con lascados alternantes (de talla bifacial) y núcleos poliédricos (de talla multifacial). Dicha variabilidad evidencia que los talladores conceptualizaron, manipularon y redujeron los recursos líticos de diferentes formas.

Finalmente, teniendo en cuenta estos procedimientos de extracción de formas-base, se postula que las mismas habrían seguido distintas trayectorias de acuerdo a un orden secuencial y/o terminal de producción (Ericson 1984). Así, en POZAC, los productos obtenidos podrían haber sido transportados en forma de nódulos y núcleos, lascas de diferentes tamaños sin modificación o con algún grado de transformación y artefactos terminados -o parcialmente terminados- hacia sitios residenciales y campamentos logísticos de Punta de la Peña y Quebrada Seca, localizados a una distancia de entre cinco y dos kilómetros aproximadamente de la cantera (Aschero et al. 2002-2004; Hocsman 2006; Martinez 2003; Pintar 1996; entre otros).

\section{Formatización de instrumentos en POZAC: bifaces en proceso de manufactura $y$ artefactos confeccionados por retalla y retoque marginal}

Los conjuntos de artefactos formatizados hallados en las ADT de POZAC permiten efectuar una aproximación a los tipos de instrumentos que se habrían confeccionado en los contextos de aprovisionamiento. Por un lado, se registran bifaces en proceso de manufactura que se relacionarían con los procedimientos iniciales de la confección de toolkits para la caza, dado el hallazgo de puntas de proyectil en sitios residenciales y logísticos de la localidad de Punta de la Peña y Quebrada Seca. En estos últimos, distintos tipos de puntas de proyectil manufacturadas sobre bifaces de vulcanitas disponibles en POZAC fueron datadas entre el 8.000 a.p. y 4.300 a.p. aproximadamente (Martinez 2003; Hocsman 2006). En efecto, en POZAC se habría desarrollado las etapas iniciales de la confección de estos tipos de instrumentos, para luego ser transportados a otros sitios donde se habría efectuado la manufactura final y/o uso (Aschero 1986; Hocsman 2006; Martinez 2003; Pintar 1996).

Por otro lado, el hallazgo de bifaces en proceso de manufactura aporta información relevante desde el punto de vista cronológico. En este sentido, el adelgazamiento bifacial y el registro consecuente de bifaces está presente en las bases residenciales de Punta de la Peña y Quebrada Seca con fechas absolutas entre los 10.000 a.p. y anteriores a los 3.000 a.p., en contextos cazadores-recolectores y en transición a la producción de alimentos (Hocsman 2006; Martínez 2003; Pintar 1996). Además, se registra una virtual ausencia del adelgazamiento bifacial y de los bifaces en bases residenciales del área con fechas posteriores al 2.000 a.p. (Hocsman 2006) (para mayor información ver Bobillo y Hocsman 2015). De este modo, la presencia de bifaces en los contextos líticos de POZAC permite postular que la cantera fue explotada, al menos, durante el Holoceno Temprano y/o Medio.

Los conjuntos de artefactos formatizados que presentan un trabajo no invasivo sobre las caras (Hocsman 2006) se encuentran confeccionados por retalla y retoque marginal. Ejemplo de ello son los cepillos, raspadores, raederas, entre otros. Al respecto, es preciso considerar que en un trabajo previo, en ADT 1 (Bobillo 2017), se registraron artefactos formatizados con este tipo de inversión de trabajo (un cortante, un cuchillo, choppers, entre otros). A partir de este hallazgo se planteó la hipótesis de que actividades de procesamiento de recursos se podrían haber 
realizado en los contextos de aprovisionamiento (Bobillo 2017; Bobillo y Hocsman 2015).

Considerando esta última afirmación, los artefactos formatizados hallados en las ADT podrían haber seguido distintas trayectorias desde el punto de vista de su elaboración, transporte y uso final. En este sentido, los instrumentos manufacturados serían el resultado de un proceso de "preparación" que implicó la elaboración de un objeto acorde a un diseño y planificación particular (Doleman 2005). En sitios residenciales de la localidad de Punta de la Peña y Quebrada Seca se hallaron artefactos confeccionados por retalla y retoque marginal que habrían sido utilizados para actividades de procesamiento de distintos recursos (Aschero et al. 1993-1994; Hocsman 2006; Pintar 2014; Toselli 1998; entre otros). Estos instrumentos se podrían haber manufacturado en sitios con disponibilidad de materias primas, como POZAC, y luego ser transportados (terminados o parcialmente manufacturados). Alternativamente, dicho instrumental podría haber sido empleado en actividades de procesamiento de recursos (posiblemente mediante acciones de corte, raspado, desbaste e incisión según funciones inferidas por Aschero 1975,1983) desarrolladas en la cantera misma. Esta hipótesis surge de considerar la posibilidad de que en las canteras se realizaran otros tipos actividades diferentes a las del aprovisionamiento estrictamente (Bobillo y Hocsman 2015; Bobillo 2017; Colombo 2013; Doelman 2005; Frank et al. 2007; Funk 2004; Hermo 2009; Messineo y Barros 2015; entre otros).

En futuras investigaciones se buscará expandir el conocimiento sobre las actividades desarrolladas en otros sectores de POZAC, relacionadas con el aprovisionamiento de recursos y con la formatización (y posible uso) de artefactos. Además, se indagarán aspectos vinculados con la cronológica de los contextos líticos, poniendo en relación los hallazgos en la cantera con datos de sitios residenciales y de otras fuentes de aprovisionamiento inmediatas a POZAC.

Para concluir, en las canteras, el accionar que los talladores desarrollan sobre el mundo material implica determinadas estrategias tecnológicas que permiten reducir y transformar los recursos líticos de acuerdo a objetivos, planes y procedimientos particulares. En este marco, los individuos y grupos de individuos desarrollan procesos de selección, producción y logística como parte de las actividades de extracción de materias primas (Bloxam y Heldal 2008), lo que genera un registro lítico diverso y significativo desde el punto de vista de la variabilidad de prácticas tecnológicas y sociales desplegadas en torno al aprovisionamiento de recursos.

Agradecimientos: A Carlos Aschero y Salomón Hocsman por los aportes realizados durante el análisis de los conjuntos líticos y por sus sugerencias e ideas para la realización de este trabajo. A Fernando Villar, Gustavo Spadoni y Ariel Escobedo por su colaboración con las actividades en el sector occidental de POZAC. A Mariano Colombo por la bibliografía y por sus comentarios. A Paz Pompei por sus recomendaciones. A los evaluadores por sus comentarios y sugerencias. Este trabajo se realizó en el marco de los proyectos PIUNT G503 y PIPCONICET 577 (dirigido por Carlos Aschero). El contenido de este trabajo es mera responsabilidad del autor.

\section{Referencias Citadas}

Aschero, C.A. 1975. Ensayo para una clasificación morfológica de artefactos líticos aplicada a estudios tipológicos comparativos. Informe al CONICET, Buenos Aires. Ms. Manuscrito en posesión del autor.

Aschero, C.A. 1983. Ensayo para una clasificación morfológica de artefactos líticos aplicada a estudios tipológicos comparativos. Apéndices A-C. Revisión. Cátedra de Ergología y Tecnología (FFyL - UBA), Buenos Aires. Manuscrito en posesión del autor.

Aschero, C.A. 1986. Estudio antropológico integral de una región de la puna Argentina: Antofagasta de la Sierra. Informe de avance. Área de arqueología. Asentamientos cazadores recolectores. Instituto Nacional de Antropología. Buenos Aires. Manuscrito en posesión del autor.

Aschero, C.A. 1988. Arqueología precerámica de Antofagasta de la Sierra. Quebrada Seca: una localidad de asentamiento.
Informe al CONICET. Carrera del Investigador Científico. Periodo 1986/87. Buenos Aires. Manuscrito en posesión del autor.

Aschero, C.A., P.S. Escola, S. Hocsman y J. Martínez 20022004. Recursos líticos en la escala microrregional Antofagasta de la Sierra, 1983-2001. Arqueología 12:9-36.

Aschero, C.A. y S. Hocsman 2004. Revisando cuestiones tipológicas en torno a la clasificación de artefactos bifaciales. En Temas de Arqueología. Análisis Lítico, editado por M. Ramos, A. Acosta y D. Loponte, pp. 1-25. Universidad Nacional de Luján, Luján.

Aschero, C.A., L. Manzi y A. Gómez 1993-1994. Producción lítica y uso del espacio en el nivel 2b4 de Quebrada Seca 3. Relaciones de la Sociedad Argentina de Antropología XIX:191-214.

Bellelli, C. 1991. Los desechos de talla en la interpretación arqueológica. Un sitio de superficie en el Valle de Piedra Parada (Chubut). Shincal 3 (2):79-93. 
Bloxam, E. y T. Heldal 2008. Identifying heritage values and character-defining elements of ancient quarry landscapes in the Eastern Mediterranean: An integrated analysis. Quarry Scapes 8, Delivery $\mathrm{N}^{\circ}$ 10. European Union.

Bobillo, F.M. 2015. Aprovisionamiento de recursos líticos: reducción de núcleos y extracción de formas-base en canteras de vulcanita (Antofagasta de la Sierra - Catamarca). La Zaranda de Ideas. Revista de Jóvenes Investigadores en Arqueología 13:9-24.

Bobillo, F.M. 2017. Estudio comparativo de Zonas de Aprovisionamiento y Cantera(ZAC) de Punta de la Peña (Antofagasta de la Sierra, Catamarca): análisis de las actividades de talla en una cantera y cantera-taller. Intersecciones en Antropología 18:67-77.

Bobillo, F.M. y S. Hocsman 2015. Mucho más que solo aprovisionamiento lítico: actividades en canteras y prácticas sociales en las fuentes de Pampa Oeste, Quebrada Seca y Punta de la Peña (Antofagasta de la Sierra, Catamarca). Revista del Museo de Antropología 8:23-44.

Carbonelli, J.P. 2015 Coleccionando miradas: aportes sobre la interpretación de los sitios de superficie de la "industria ampajanguense". Revista del Museo de Antropología 8:7-22.

Colombo, M. 2013. Los Cazadores y Recolectores Pampeanos y sus Rocas. La Obtención de Materias Primas Líticas Vista Desde las Canteras Arqueológicas del Centro de Tandilia. Tesis para optar al título de Doctor en Ciencias Naturales. Facultad de Ciencias Naturales y Museo, Universidad Nacional de La Plata, Argentina.

Church, T. 1995. Terms in lithic resource studies. Lithic resource studies: a source for archaeologist. Special Publication, Department of Anthropology. Lithic Technology 3:9-25.

Crabtree, D.E. 1972. An introduction to flintworking. Occasional Papers of the Idaho State University Museum 28:1-98.

Doelman, T. 2005. Tool time: The influence of tool manufacture, use, and discard on the formation and composition of a quarry assemblage. Lithic Technology 30:13-26.

Ericson J.E. 1984. Toward the analysis of lithic production systems. En Prehistoric Quarries and Lithic Production, editado por J.E. Ericson y B.A. Purdy, pp 1-9. Cambridge University Press, Cambridge.

Escola, P.S. 1993. De percusión y percutores. Palimpsesto 3:33-51.

Escola, P. 2002. Disponibilidad de recursos líticos y fuentes de aprovisionamiento en un sector de la puna meridional. Mundo de Antes 3:65-86.

Flenniken, J.J. y J.P. White 1985. Australian flaked stone tools: a technological perspective. Records of the Australian Museum 36 (3):131-151.

Funes Coronel J.A. y J.G. Martínez 2013. Lithic production sequences in the southern Argentinian Puna during the initial middle Holocene. Quarry-workshop characterization in the mid-course Ilanco river. Quaternary International 307:74-80.

Frank, A.D., F. Skarbun y M.F. Paunero 2007. Hacia una aproximación de las primeras etapas de reducción lítica en el Cañadón de la Mina, Localidad Arqueológica La María, Meseta Central de Santa Cruz, Argentina. Magallania 35:133-144.

Funk R.E. 2004. An ice age quarry-workshop: The West Athens Hills Site revisited. New York State Museum Bulletin 504. The University of New York, State of Education Department Albany, New York.

González Bonorino, F.y M. Teruggi 1952.Léxico sedimentológico. Instituto Nacional de Investigación de las Ciencias Naturales y
Museo Argentino de Ciencias Naturales "Bernardino Rivadavia". Publicación de Extensión Cultural y Didáctica 6. Buenos Aires.

Goren-Inbar, N., L. Grosman y G. Sharon 2011. The technology and significance of the Acheulian giant cores of Gesher Benot Ya'aqov, Israel. Journal of Archaeological Science 38:1901-1917.

Haury, C.E. 1994. Defining lithic procurement terminology. Lithic Technology 3:26-32.

Hermo, D.O. 2009. Estructura de los recursos líticos y paisajes arqueológicos en el Nesocratón del Deseado (Santa Cruz, Argentina). Arqueología Sudamericana 5 (2):179-203.

Hocsman, S. 2006. Producción Lítica, Variabilidad y Cambio en Antofagasta de la Sierra (5500-1500AP). Tesis para optar a grado de Doctor en Ciencias Naturales. Facultad de Ciencias Naturales y Museo, Universidad Nacional de La Plata, Argentina.

Inizan, M.L., M. Reduron-Ballinger, H. Roche y J. Tixier 1999. Introduction. En Technology and Terminology of Knapped Stone. Préhistoire de la Pierre Taillée, editado por M.L. Inizan, M. Reduron-Ballinger, H. Roche y J. Tixier, pp. 13-17. CREP, Nanterre.

Martín Blanco, P., A. Jimenez Manzanares, J. Sanguino González y A.J. Gómez Laguna 1994. Identificación de cadenas operativas líticas en el sitio arqueológico de "Xasa de la Mina II" (Argamasilla de Alba, c. Real). Consideraciones acerca de los yacimientos superficiales sin contexto estratigráfico. Zephyrvs XLVII:15-40.

Martínez, J. 2003. Ocupaciones Humanas Tempranas y Tecnología de Caza en la Microrregión de Antofagasta de la Sierra (100007000 AP). Tesis para optar al grado de Doctor en Arqueología. Facultad de Ciencias Naturales e Instituto Miguel Lillo, Universidad Nacional de Tucumán, Argentina.

Messineo, P.G. y M.P. Barros 2015. Lithic raw materials and modes of exploitation in quarries and workshops from the center of the pampa grasslands of Argentina. Lithic Technology 40:3-20.

Nami, H.G. 1992. El subsistema tecnológico de la confección de instrumentos líticos y la explotación de los recursos del ambiente: una nueva vía de aproximación. Shincal 2:33-53.

Nelson, M.C. 1991. The study of technological organization. En Archaeological Method and Theory, editado por M.B. Schiffer, pp. 57-100. The University of Arizona Press, Tucson.

Pintar, E. 1996. Prehistoric Holocene Adaptations to the Salt Puna of Northwestern Argentina. Tesis para optar al título de Doctora, Graduate Faculty of Dedman College, Southern Methodist University, Texas, Estados Unidos.

Pintar, E. 2014. Continuidades e hiatos ocupacionales durante el Holoceno Medio en el borde oriental de la Puna Salada, Antofagasta de la Sierra, Argentina. Chungara Revista de Antropología Chilena 46:51-72.

Somonte, C. 2005. Uso del espacio y producción lítica en Amaicha del Valle (Departamento Tafí del Valle, Tucumán). Intersecciones en Antropología 6: 43-58.

Somonte, C. y C. Baied 2011. Recursos líticos, aprovisionamiento $\mathrm{y}$ aspectos temporales de fuentes de abastecimiento en Amaicha del Valle, Tucumán, Argentina. Comechingonia 14:97-113.

Tchilinguirian, P. 2008. Paleoambientes Holocénicos en la Puna Austral, Provincial de Catamarca (27ㅇ): Implicancias Geoarqueológicas. Tesis para optar por el grado de Doctor. Departamento de Ciencias Geológicas, Facultad de Ciencias Exactas y Naturales, Universidad de Buenos Aires, Argentina. 
Toselli,A. 1998. Selección de Materias Primas Líticas y Organización Tecnológica en el Sitio Punta de la Peña 4 (PP4), Depto. Antofagasta de la Sierra, Prov. de Catamarca. Tesis para optar por el título de Arqueóloga. Facultad de Ciencias Naturales e Instituto Miguel Lillo, Universidad Nacional de Tucumán, Argentina.
Toth, N., J. Clark y G. Ligabue 1992. The last stone axemakers. Scientific American 267:88-93.

Whittaker, J. 1994. Flintknapping: Making and Understanding Stone Tools. University of Texas Press, Austin.

\section{Notas}

1 Las rocas ígneas presentes en la microrregión de Antofagasta de la Sierra se diferencian en base a la proporción de vidrio, pudiéndose identificar distintos grupos. En este sentido, el término vulcanita se emplea con base en la propuesta de unificación de denominaciones de materias primas líticas realizada por Aschero et al. (2002-2004).
2 El Número Mínimo de Desechos (NMD) refiere a la totalidad de lascas enteras y fracturadas con talón presentes en la muestra (Aschero et al. 1993-1994). Según los autores, este tipo de piezas proporcionan una representación más exacta entre el producto de talla y la actividad. 